\title{
LASER CHEMISTRY IN GREECE
}

Research activities in laser chemistry appeared in Greece first in the 1970's, with the theoretical work of the Theoretical and Physical Chemistry Institute at the National Hellenic Research Foundation (NHRF) in Athens playing a prominent role. Experimental studies were particularly promoted and developed in the early 1980's in Crete, with the establishment of the research programme of the Institute of Electronic Structure and Laser of the Foundation for Research and Technology-Hellas (FORTH). Similar activities were also undertaken by experimental groups active at the NHRF, the Technical University of Athens and the Universities of Ionnina, Crete and Patras. It should be noted that a large part of the fundamental studies carried out particularly at FORTH in Crete, were eventually linked to European initiatives of technological research and were supported in part by European Community R\&D programmes, such as the BRITE-EURAM programme and the EUREKAEUROLASER project. The required inter-european and/or industrial collaboration within the context of these programmes is reflected in some of the articles appearing in this issue. The establishment of the "Ultraviolet Laser Facility" at FORTH in 1990, under the auspices of the "Large Installations Plan" of the European Community has played a key role in further promoting and establishing such collaborative efforts.

This issue includes articles representing research work which may be categorized into three major sections: a) Laser chemistry induced in the IR and UV, b) Studies on coherent sources emitting at short wavelengths (UV and VUV) and c) Spectroscopic studies of analytical interest.

The establishment of a critical mass of scientists active in the field of laser chemistry, when combined with emphasis in scientific quality and technological relevance ensures an optimistic outlook for the future of this field in Greece.

C. Fotakis

Guest Editor 